\title{
Conjetura y demostración en el aula en la formación de docentes*
}

Edgar Balaguera Ascencio ${ }^{1}$

Recibido: 25-02-2020

Aceptado: 04-03-2020

\section{Resumen}

Este trabajo de investigación muestra cómo los procesos de conjetura y demostración matemática en el marco de la resolución de problemas favorecen el desarrollo del pensamiento matemático manifestado por los estudiantes de la Licenciatura en Educación Básica con énfasis en Matemáticas de la Universidad Santo Tomás, en la modalidad a distancia. La metodología de esta investigación fue de carácter cualitativo, a través de estudios de caso de los participantes, y se desarrolló bajo los parámetros de la ingeniería didáctica de Michèle Artigue. El marco teórico se fundamenta en los aportes de Gila Hanna (2000) en torno a la funcionalidad de la demostración matemática, los aportes de Harel y Sowder (2007) respecto a los esquemas de demostración desarrollados por los estudiantes y la resolución de problemas desde la perspectiva de Alan Schoenfeld (1985).

Palabras clave: demostración matemática, conjetura, razonamiento abductivo, resolución de problemas, formación de docentes.

\footnotetext{
* Artículo de investigación científica resultado de la tesis doctoral.

1. Doctor en Educación Matemática, Universidad Antonio Nariño, Bogotá, Colombia. Docente de matemáticas del Departamento de Ciencias Básicas, Universidad Santo Tomás, Bogotá, Colombia; grupo de investigación Ciencia e Ingeniería de Materiales $(\mathrm{CIM})$, línea de investigación de educación y pedagogía de las ciencias básicas.

Correo electrónico: edgarbalaguera@usantotomas.edu.co

ORCID: https://orcid.org/0000-0002-9501-8320

Google Scholar: https://scholar.google.es/citations?hl=en\&pli=1 \&user=TgfS-fkAAAAJ CvLac: https://scienti.colciencias.gov.co/cvlac/visualizador/generarCurriculoCv.do?.cod_ $\mathrm{rh}=0001587357$
} 


\title{
Conjecture and classroom demonstration in teacher training
}

\begin{abstract}
This research work shows how the processes of conjecture and mathematical demonstration in the framework of problem solving favor the development of mathematical thinking manifested by students of the Bachelor of Basic Education with an emphasis on Mathematics of the Santo Tomás University, in the modality from distance. The methodology of this research was qualitative in nature, through case studies of the participants, and was developed under the parameters of the didactic engineering of Michèle Artigue. The theoretical framework is based on the contributions of Gila Hanna (2000) regarding the functionality of the mathematical proof, the contributions of Harel and Sowder (2007) regarding the demonstration schemes developed by the students and the resolution of problems from the Alan Schoenfeld's perspective (1985).
\end{abstract}

Keywords: mathematical proof, conjecture, abductive reasoning, problem solving, teacher training.

\section{Introducción}

La formación de docentes es un tema que siempre estará en discusión debido a los cambios sociales, históricos, culturales, políticos y tecnológicos. Los nuevos docentes de matemáticas deben estar al día en materia de pedagogía, matemáticas y tecnología para abordar de manera pertinente las potencialidades, las deficiencias y problemáticas del sistema educativo. Luego, la formación del docente se convierte en un ingrediente esencial para la enseñanza y aprendizaje de las matemáticas a futuras generaciones de estudiantes. La enseñanza de las matemáticas parece estar 
coaccionada en función de las creencias o enfoques establecidos durante el proceso de formación del maestro. Es de vital importancia que las nuevas generaciones de maestros transformen desde los primeros niveles de la educación el papel del razonamiento, la conjetura y la demostración matemática en el aula hacia una nueva perspectiva más falibilista.

Al analizar recientes investigaciones respecto a la conjetura y demostración matemática se encuentra que existe una preocupación general respecto a cómo los estudiantes y docentes abordan el papel de la demostración en el aula. Esto se debe a que, en su mayoría, predominan los esquemas de demostración empírica en todos los niveles y en muchos casos una ausencia total de los métodos de demostración deductiva.

Existe un reconocimiento internacional de la importancia del razonamiento y la demostración en el aprendizaje de las matemáticas de los estudiantes en todos los niveles de educación y de las dificultades con el razonamiento, construcción y comprensión de los argumentos matemáticos que cumplen con el estándar de demostración. Los maestros también enfrentan dificultades con el razonamiento y la demostración y los materiales existentes tienden a ofrecer un apoyo inadecuado para el trabajo en el aula. Todos ellos describen el razonamiento y la demostración como importantes, pero difíciles de enseñar y aprender. (Harel, Stylianides, Boero, Miyazaki y Reid, 2017, p. 459). Recientes estudios muestran que existe una necesidad de seguir investigando en este campo y encontrar respuestas a preguntas abiertas tales como: ¿qué intervenciones en el aula pueden hacer que los estudiantes vean la necesidad de mejorar sus esquemas de demostración empíricas y autoritarias en esquemas de demostración deductiva?, ¿cómo se construyen, verifican y aceptan las demostraciones en el aula?, ¿cuál es el rol de la tecnología en la enseñanza-aprendizaje de la conjetura y la demostración matemática?, ¿cómo la concepción de la demostración a lo largo de la historia de las matemáticas puede influir positivamente en el proceso de enseñanza-aprendizaje de la misma? 
Al hacer un balance de las investigaciones en torno a la funcionalidad de la demostración, Stylianides y Stylianides (2017) señalan que existen marcos teóricos bien fundamentados que sustentan los obstáculos o dificultades en torno a su enseñanza y aprendizaje ya que, en cualquier nivel educativo, las creencias en torno a la demostración muestran que es un proceso difícil de enseñar y difícil de asimilar por parte de los estudiantes. Existe una buena base de investigaciones en torno a cómo los estudiantes entienden (erróneamente) la demostración, pero no hay evidencia de intervenciones positivas de este proceso en el aula.

Stylianides y Stylianides (2017) establecen que en los próximos 15 o 20 años las investigaciones deben girar en torno a la construcción de un marco teórico sólido sobre las formas en que la instrucción puede mejorar el aprendizaje de la demostración en el estudiante. Durante las últimas décadas los estudios se han enfocado en los niveles de educación superior. Por eso es importante, como lo manifiestan los autores, que en los próximos años las investigaciones también se centren alrededor del diseño de intervenciones en el aula respecto a la enseñanza y el aprendizaje de la demostración en los niveles primarios, ya que desde un punto epistemológico, estas investigaciones pueden ayudar a lograr una mayor continuidad en las formas de pensar de los estudiantes en diferentes niveles de educación y disminuir la brecha existente en muchos países entre primaria y la introducción abrupta de la demostración en los estudiantes de la escuela secundaria.

Este trabajo tiene como objetivo la profundización teórica y la caracterización de los procesos de conjetura y demostración en el aula manifestados por los estudiantes de la Licenciatura en Educación Básica con énfasis en Matemáticas de la Universidad Santo Tomás en la modalidad a distancia, y responde al siguiente problema de investigación: ¿cómo la conjetura y la demostración matemáti$\mathrm{ca}$, en el marco de la resolución de problemas, favorecen el desarrollo del pensamiento matemático de los docentes en formación? 


\section{Metodología}

La metodología de esta investigación es de tipo cualitativa, ya que permite al investigador analizar y comprender la naturaleza científica de los fenómenos que emergen desde la perspectiva de los participantes en un determinado contexto. El método aplicado para esta investigación es el estudio de casos, dado que es posible investigar empíricamente mediante procedimientos inductivos y deductivos los aspectos subjetivos y objetivos de la vida social tal como lo señalan Neiman y Quaranta (2012). Este estudio de casos se aborda desde la ingeniería didáctica (ID) de Michèle Artigue (1995), quien la define como una metodología de investigación que aborda de manera experimental las generalidades de la concepción, la realización, la observación y el análisis de las secuencias de enseñanza desde cuatro fases: el análisis preliminar, la concepción y análisis a priori de las situaciones didácticas, la experimentación y el análisis a posteriori, y la validación.

Como estrategia en el diseño de las secuencias de aprendizaje se utilizó el enfoque centrado en la resolución de problemas desde la perspectiva de Alan Schoenfeld (1985) que aborda este proceso desde cuatro factores: los recursos, las heurísticas, el control y el sistema de creencias.

\section{Población y muestra}

Esta investigación se desarrolló en la Licenciatura en Educación Básica con énfasis en Matemáticas de la Universidad Santo Tomás en la modalidad a distancia. La Universidad Santo Tomás ofrece a través de sus centros de atención universitaria (CAU) programas de educación a distancia con presencia en 23 municipios o ciudades del país. En esta investigación participaron 6 estudiantes de séptimo semestre que se encontraban inscritos en la asignatura de Número y Cantidad; la modalidad es totalmente a distancia y se desarrolló a través de la plataforma Moodle. 
Tabla 1. Población y muestra.

\begin{tabular}{|c|c|c|c|}
\hline Identificación & $\begin{array}{c}\text { Centro de atención } \\
\text { Universitario (CAU) }\end{array}$ & Edad & Sexo \\
\hline Estudiante 1 & Barrancabermeja & 22 & Femenino \\
\hline Estudiante 2 & Bogotá & 25 & Femenino \\
\hline Estudiante 3 & Chiquinquirá & 22 & Masculino \\
\hline Estudiante 4 & Ocaña & 23 & Masculino \\
\hline Estudiante 5 & Montería & 22 & Femenino \\
\hline Estudiante 6 & Armenia & 23 & Femenino \\
\hline
\end{tabular}

Fuente: elaboración propia

Los estudiantes participaron mediante encuentros virtuales a través de videoconferencias en el horario de asesorías establecidos por la universidad o en otros horarios acordados con algunos estudiantes, dada la flexibilidad que ofrece la modalidad a distancia. También participaron en foros de discusión, presentaron videos de los trabajos entregados y el envío de los archivos con el desarrollo de las actividades. Los estudiantes inscritos en esta licenciatura han elegido la dinámica de la educación a distancia fundamentalmente por la flexibilidad en los horarios, los encuentros asincrónicos y las barreras de distancia de la educación presencial. La mayoría de estos estudiantes actualmente se encuentran trabajando en regiones de difícil acceso o alejados de un centro de educación universitario presencial. Bajo estas condiciones, la educación a distancia se convierte en una solución de cobertura en educación que permite suplir las limitaciones de la educación presencial.

Debido a las condiciones de aislamiento en que se encuentran estos estudiantes, su interacción con procesos de pensamiento matemático es baja y algunos de ellos desconocen la importancia de los procesos de conjetura y demostración en el aula. El campo profesional de estos maestros en formación será la enseñanza de las matemáticas en los primeros niveles de educación y en muchas ocasiones completarán su carga académica con las otras áreas del ciclo de primaria. Actualmente en Colombia la enseñanza de las matemáticas en los niveles de primaria es asumida por docentes que deben tener un dominio de todas las áreas. Por eso el impacto de esta investigación trasciende más allá de esta muestra, ya que, 
siendo maestros en formación, en un futuro serán multiplicadores de este enfoque de la demostración matemática.

A continuación, se hace una descripción detallada del desarrollo metodológico de la investigación, que incluye los análisis preliminares, la concepción y el análisis a priori, la experimentación y el análisis de los datos, el análisis a posteriori, y la validación.

\section{Análisis preliminares}

Debido a la poca importancia que actualmente reciben los procesos de demostración en el aula, en las últimas décadas se han incrementado notablemente las investigaciones en torno a este tema. En los años setenta y finales del siglo pasado, las investigaciones giraban en torno a la identificación de las dificultades que estudiantes y maestros manifestaban respecto a la construcción de demostraciones matemáticas vistas desde la axiomatización o formalización de ellas. En la actualidad las investigaciones giran en torno a la implementación de prácticas positivas que destaquen la importancia de estas actividades en el desarrollo del pensamiento matemático. A continuación, se relacionan las problemáticas más frecuentes en torno a la demostración matemática manifestada por docentes y estudiantes. Este análisis se realiza desde las dimensiones propuestas en la ID.

Tabla 2. Dimensiones del análisis preliminar.

\begin{tabular}{|l|l|}
\hline \multicolumn{1}{|c|}{ Dimensión } & \multicolumn{1}{c|}{ Problemáticas } \\
\hline Epistemológica & $\begin{array}{l}\text { - Se subestima la capacidad del estudiante. } \\
\text { - Proceso difícil de enseñar y aprender. } \\
\text { - Su finalidad es convencer. } \\
\text { - Enfoque es de tipo tradicional (formal, autoritario, ritual). }\end{array}$ \\
\hline Cognitiva & $\begin{array}{l}\text { - Ritual sin sentido. } \\
\text { - Memorización y reproducciones literales. } \\
\text { - Solo construyen esquemas de demostración empíricos. }\end{array}$ \\
\hline Didáctica & $\begin{array}{l}\text { - No está incluido en el currículo (fue eliminado). } \\
\text { - Carencia de material didáctico y tecnológico. } \\
\text { - Se presenta como un producto. } \\
\text { - Poca o ninguna formación en los maestros potenciales. }\end{array}$ \\
\hline
\end{tabular}

Fuente: elaboración propia 


\section{La concepción y el análisis a priori}

Esta fase se diseñó teniendo en cuenta la hipótesis, los objetivos, el estado del arte y el marco teórico de la investigación. A continuación de describen las variables macrodidácticas y microdidácticas que intervienen, y se especifica la forma en que se aborda los procesos de conjetura y de demostración matemática en el aula.

Las variables macrodidácticas describen el enfoque con el que se aborda la conjetura y la demostración en el aula en esta investigación, mientras que las variables microdidácticas determinan la forma en que se abordan y se diseñan las secuencias de aprendizaje.

Tabla 3. Variables en la concepción y el análisis a priori.

\begin{tabular}{|l|l|}
\hline \multicolumn{1}{|c|}{ Variables macrodidácticas } & \multicolumn{1}{c|}{ Variables microdidácticas } \\
\hline $\begin{array}{l}\text { •Funcionalidad de la demostración en el aula } \\
\text { (Gila Hanna). }\end{array}$ & $\begin{array}{l}\text {-Razonamiento abductivo (Charles Sanders } \\
\text { Peirce) }\end{array}$ \\
$\begin{array}{l}\text {-Modelo de formación de docentes de Fennema } \\
\text { y Franke }\end{array}$ & $\begin{array}{l}\text { Resolución de problemas retadores (Mary Falk } \\
\text { de Losada) } \\
\text {-Perspectiva integral de la demostración y } \\
\text { esquemas de demostración (Harel y Sowder) }\end{array}$ \\
$\begin{array}{l}\text {-Resolución de problemas (Alan Schoenfeld) } \\
\text { Campbell) }\end{array}$ \\
•Educación a distancia.
\end{tabular}

Fuente: elaboración propia

Tomando como referente cada uno de los aspectos mencionados, se plantearon las siguientes hipótesis globales y locales.

\section{Hipótesis global}

La conjetura y demostración matemática en el marco de la resolución de problemas retadores favorecen el desarrollo del pensamiento matemático en los estudiantes de la Licenciatura en Educación Básica con énfasis en Matemáticas de la Universidad Santo Tomás en la modalidad a distancia. 


\section{Hipótesis locales}

- La interacción constante con situaciones matemáticas retadoras mediante la exploración, la manipulación de los objetos matemáticos y los recursos con los que dispone permiten desarrollar procesos de elaboración de hipótesis y formulación de conjeturas.

- El desarrollo del razonamiento inductivo, abductivo y deductivo en el marco de la resolución de problemas favorece el desarrollo deductivo de esquemas de demostración con miras a mejorar sus definiciones, construir significados más robustos y profundizar teóricamente sus resultados.

\section{La experimentación y el análisis de datos}

En esta etapa se diseñaron las secuencias de aprendizaje teniendo como referentes las variables micro y macrodidácticas y se abordaron desde tres fases (exploración, validación y demostración). Ya que la modalidad de la asignatura es a distancia, esta se desarrolló a través de la plataforma Moodle. Esta plataforma cuenta con aulas virtuales en las que se encuentran las unidades temáticas, los foros de discusión, los encuentros virtuales denominados tutorías (a través de videoconferencias) y los enlaces para entregar y cargar los archivos de las actividades correspondientes.

El análisis de datos se realizó teniendo en cuenta los parámetros y características de la teoría fundamentada. Este proceso se hace mediante una descripción de lo que sucede al interior de cada secuencia de aprendizaje, un ordenamiento sistemático mediante propiedades, categorías y dimensiones, hasta finalmente llevarlo a un estado de teorización derivada de los datos.

La codificación sistemática de los datos se realizó a través del software Atlas.ti 8. Este programa es una poderosa herramienta para el análisis cualitativo de los datos que permite al investigador encontrar, descubrir y clasificar códigos a través de citas de 
textos, videos, audios, imágenes, etc., con el objetivo de atrapar la realidad mediante redes conceptuales, tal como lo señala la teoría fundamentada. En total se realizaron 6 secuencias de aprendizaje con la misma estructura. A continuación, se muestra un ejemplo de las secuencias de aprendizaje desarrolladas por cada uno de los estudiantes.

\section{Problema banda de colores}

Una cuadrícula de tamaño $123 \times 123$ se desea pintar utilizando 6 colores tal como se muestra en la siguiente figura.

Figura 1. Banda de colores.

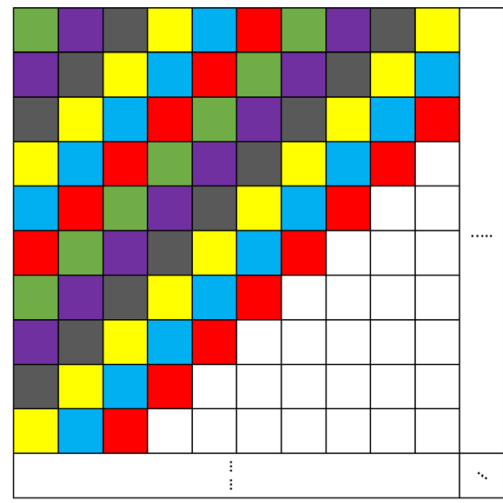

Fuente: elaboración propia

Se quiere determinar qué color se utilizó más que los demás y la cantidad de cuadrículas de cada color.

\section{Fase exploratoria}

En esta fase se analizan las estrategias desarrolladas por los seis estudiantes para resolver el problema de la banda de colores en una cuadrícula de $123 \times 123$, los estudiantes utilizaron cuadrículas más pequeñas con el propósito de encontrar regularidades. Este método de utilizar casos más pequeños se da por la imposibilidad de trabajar con una cuadrícula tan grande. Al utilizar casos más pequeños la mayoría de los estudiantes realizaron descomposiciones 
de la cuadrícula con la finalidad de facilitar el conteo de colores e identificar cuál color se utilizó más. A continuación se redactan de modo general las conjeturas encontradas por los estudiantes y sus mecanismos de validación.

Conjetura 1. Si la cantidad de colores es divisor del área de la cuadrícula, entonces la cuadrícula contiene la misma cantidad de colores.

Conjetura 2. Si la cantidad de colores es divisor de la longitud de la cuadrícula, entonces la cuadrícula contiene la misma cantidad de colores.

Conjetura 3. Si una cuadrícula es de tamaño $\mathrm{n} \times \mathrm{n}$, entonces el color que más se utiliza, está representado por el residuo de dividir la longitud de la cuadrícula entre la cantidad de colores.

\section{Fase de validación}

Como se mencionó en la fase de exploración, la mayoría de los estudiantes basaron la validación de sus conjeturas en métodos de descomposición de las cuadrícula y a partir de estas, argumentaron deductivamente los resultados encontrados. El método de descomposicion se caracteriza por tomar un caso más pequeño en el que realizan una transformación del problema.

Tabla 4. Evidencia de la fase de validación.

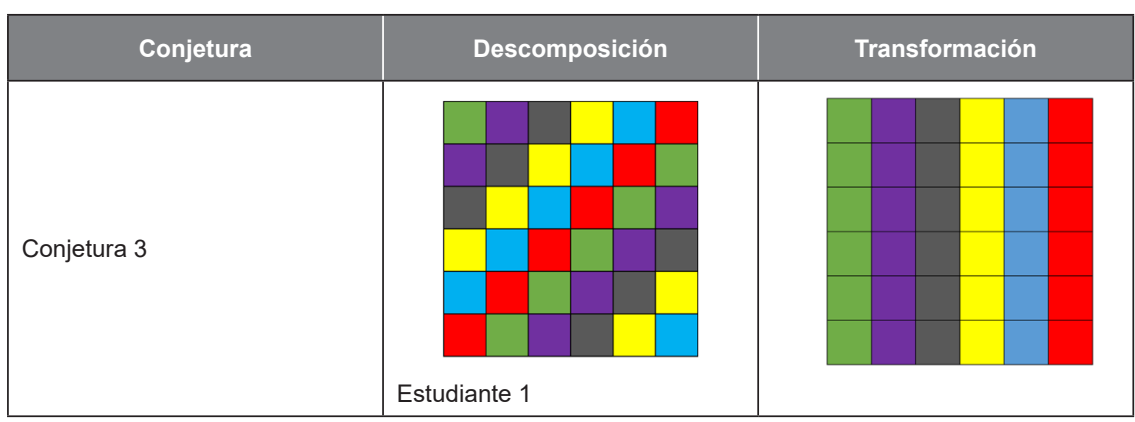

Fuente: elaboración propia 
Este proceso de descomposición y transformación consiste en dividir la cuadrícula en cuadrados mas pequeños de $6 \times 6$ y luego transformar ese cuadrados por columnas de igual color. El propósito de la transformación es dejar en evidencia una propiedad invariante; en este caso, se desea mostrar que cada cuadrado de 6 x 6 contiene 6 cuadros de cada color.

\section{Fase de demostración}

En esta fase los estudiantes describieron la solución del problema de forma general para cuadrículas de tamaño $n \times n$ y tamaño $m \times n$. A continuación, se relacionan de manera general los esquemas de demostración desarrollados por los seis estudiantes para resolver el problema de manera general.

El estudiante 6, parte la cuadrícula en cuatro secciones, de tal forma que transforma la situación matemática en un problema geométrico. Una vez establecida esta transformación el estudiante procede a demostrar la conjetura 3, aplicando un método de conteo de forma general.

Tabla 5. Evidencia de la fase de demostración.

\begin{tabular}{|c|c|c|c|c|}
\hline Conjetura & \multicolumn{3}{|c|}{ Descomposición } & Demostración \\
\hline Conjetura 3 & $\begin{array}{c}\mathrm{m} \\
\perp \\
\top \\
\mathrm{r} \\
\perp\end{array}$ & $\mathrm{mxm}$ & $r-1$ & $\begin{array}{l}\text { "Para contar la cantidad de colores de la } \\
\text { cuadricula, se divide la longitud de la cuadricula n } \\
\text { en la cantidad de colores } k \text { y se obtiene } \\
n=k m+r, m \text { es múltiplo de } n-r \text { y el residuo } \\
r \text { representa la posición del último color en la } \\
\text { longitud de la cuadricula. } \\
\text { Luego se particiona la región en cuatro partes } \\
\text { como se muestra en la figura y se calcula cada } \\
\text { parte: } \\
\text { En la región } m \times m \text {, el total de cada color es } m^{2} k \text {. } \\
\text { En cada región } m \times r \text { hay } m r \text { unidades de cada } \\
\text { color. } \\
\text { En la región } r \times r \text { hay } r \text { unidades del color que } \\
\text { ocupa esa posición y que están en la diagonal } \\
\text { principal, el número de unidades de los otros } \\
\text { colores es menor que los de la diagonal } r \text {. } \\
\text { Cantidad de colores en la posición } r \text {. } \\
C_{r}=m^{2} k+2 m r+r \text { " }\end{array}$ \\
\hline
\end{tabular}

Fuente: elaboración propia 
Los estudiantes 1, 2, 3, 4 y 5 basaron su demostración de manera deductiva a través de un ejemplo genérico a manera de descripción del método, pero sin utilizar un lenguaje algebraico.

Tabla 6. Evidencia de la fase de demostración.

\begin{tabular}{|l|l|l|l|l|l|l|}
\hline Conjetura & Conjetura 1 & & & & & \\
\hline
\end{tabular}

Fuente: elaboración propia

\section{Análisis a posteriori y validación}

La fase de validación de la ID es interna y supone una contrastación del análisis a priori con el a posteriori con el fin de validar o refutar las hipótesis planteadas en la investigación. Artigue (1995) enfatiza que debido a la formulación relativamente global de las hipótesis de investigación que suponen procesos de aprendizaje a largo plazo, la amplitud de la ID no le permite comprometerse en realidad con un proceso de validación.

En esta fase se busca mostrar evidencia de causa y efecto que dé explicación a las observaciones y resultados encontrados. El 
análisis a posteriori y la validación interna se abordarán desde las hipótesis globales y locales planteadas en el análisis a priori. Las hipótesis locales caracterizan fenómenos observados en el desarrollo de las secuencias de aprendizaje y la hipótesis global describe fenómenos generales en torno a la implementación de la conjetura y demostración en el aula en la formación de docentes. A continuación, se analizan en detalle cada una de ellas.

\section{Hipótesis locales}

\section{Hipótesis $n .^{\circ} 1$}

La interacción constante con situaciones matemáticas retadoras mediante la exploración, la manipulación de los objetos matemáticos $y$ los recursos con los que dispone permite desarrollar procesos de elaboración de hipótesis y formulación de conjeturas.

En cada una de las secuencias de aprendizaje se evidencia una motivación natural de querer resolver cada uno de los problemas. Al abordar cada situación, los estudiantes tomaron como positivo el hecho de no necesitar un alto dominio de los conceptos matemáticos; esto generó un nivel de confianza y disposición para iniciar a explorar cada problema propuesto.

En la fase de exploración de las secuencias de aprendizaje, los estudiantes comenzaron interactuando con cada problema retador. El hecho de manipular objetos matemáticos como las bandas de colores, permitió la comprensión de cada situación matemática. En la medida que cada uno de ellos comenzó a interactuar con estos objetos matemáticos que en un principio parecen caóticos y que dependen de variables hasta el momento no controladas, su dominio sobre la dinámica de las operaciones comenzó a tener sentido. Este dominio hace referencia al conocimiento previo (matemático, informal) y la forma como se aborda el problema (herramientas, técnicas).

La exploración, los recursos (los objetos, el dominio) y las operaciones sobre los objetos determinan una transición de las ope- 
raciones concretas hacia la comprensión de las situaciones matemáticas.

Figura 2. Fase de exploración.

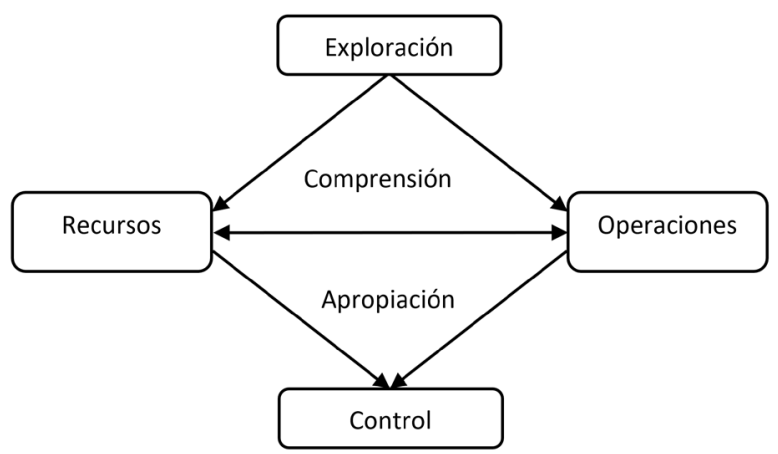

Fuente: elaboración propia

Una vez definida la comprensión del problema, la interacción entre los recursos y sus operaciones, los estudiantes enfrentan los problemas desde una nueva perspectiva y muestran un sentido de apropiación de la situación matemática abordada, lo que da como resultado un control total de la situación. En este punto, el estudiante es consciente de lo que sucede al interior de cada situación, sus decisiones comienzan a ser monitoreadas y evaluadas dependiendo de los resultados generados; es decir, el estudiante establece procesos metacognitivos de sus actuaciones.

Esta etapa de control no es inmediata. Durante el desarrollo de las secuencias se observó que algunos participantes en su fase inicial realizaban operaciones sobre los objetos, pero realmente no tenían control de la situación. Este hecho se debe a que, en la etapa de exploración, las operaciones sobre los objetos no estaban encaminadas a la comprensión sino a la manipulación de los objetos. Este tipo de situaciones, desde la metodología de investigación de la ID, se reconocen como distorsiones; estas requieren un ajuste o una reacomodación durante el desarrollo de las secuencias de aprendizaje. En cada uno de estos casos se buscó 
a través de los encuentros por videoconferencia la certeza de la comprensión de los problemas retadores. Este trabajo se hizo a través de la interacción entre ellos sin comprometer el papel del profesor dentro de la ID.

Figura 3. Proceso de la formulación de la conjetura.

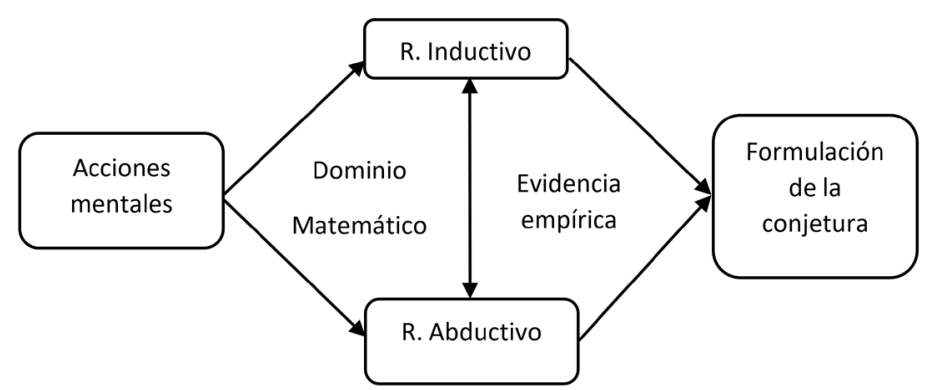

Fuente: elaboración propia

Una vez definida la etapa de control por parte de los estudiantes, las operaciones sobre los objetos comienzan a ser planificadas y se definen como acciones mentales (ver figura 3). Estas acciones están determinadas por sus razonamientos que en primera instancia son de tipo inductivo y abductivo; aquí la intuición y la sospecha sobre los hechos observados juegan un papel importante hacia el camino de la elaboración de la hipótesis. El razonamiento abductivo tiene como función descubrir hechos sorprendentes sobre las acciones realizadas y el razonamiento inductivo tiene como función la recolección y organización de la evidencia de los hechos observados. Una vez analizada esta evidencia, emerge de manera natural el proceso de formulación de la conjetura. Esta dualidad entre el descubrimiento y la inducción de los resultados está condicionada por su dominio matemático, es decir, sus mecanismos de verificación son empíricos a través gráficos, tablas de datos, ensayo y error, etc. Sus justificaciones se dan mediante la experiencia y sobre situaciones concretas; en esta etapa, todavía no se concibe un desprendimiento de lo concreto hacia lo abstracto. 
En la fase de validación sus resultados ya no dependen de la manipulación de los objetos ni sus operaciones sino del dominio matemático y la argumentación de sus afirmaciones. Es decir, existe un desprendimiento de las operaciones concretas hacia un nivel de operaciones formales: sus acciones mentales comienzan a depender del razonamiento matemático, la argumentación y el nivel de abstracción desarrollado por cada uno de ellos. Este proceso de validación se da mediante la transferencia y transformación de las propiedades invariantes inmersas en cada uno de los problemas retadores. Por transferencia se entiende que ellos identifican propiedades comunes sobre situaciones locales y las reproducen en situaciones globales del problema; por transformación se entiende que existe un proceso de manipulación y modificación de la situación matemática aparentemente compleja hasta llevarla a un estado de verificación evidente.

Figura 4. Caracterización de la fase de validación.

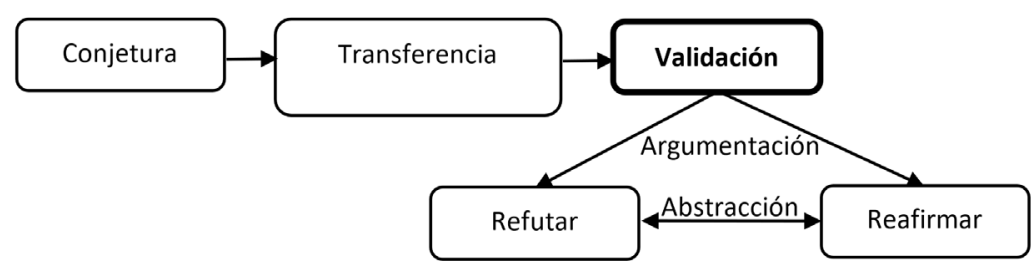

Fuente: elaboración propia

Dentro del proceso de refutación de la conjetura, se evidencia que el contraejemplo es el mecanismo que utilizan los estudiantes para objetar una conjetura incorrecta. El uso del contraejemplo les conduce a un desequilibrio cognitivo sobre los argumentos que les llevaron al planteamiento y formulación de la conjetura; su reacomodación conduce a la modificación sobre sus acciones mentales con miras a encontrar nuevos resultados respaldados por nueva evidencia empírica recolectada. En general, el hecho de encontrar un contraejemplo los lleva a hacer una revisión cuidadosa de la evidencia y las heurísticas que los llevaron al planteamiento de dicha hipótesis. Una vez detectado el hecho que generó la falsa hipótesis, se realizan los cambios respectivos y se comienza de nuevo el proceso de reformulación de la conjetura. 


\section{Hipótesis $n .^{\circ} 2$}

El desarrollo del razonamiento inductivo, el abductivo y el deductivo en el marco de la resolución de problemas favorecen el desarrollo de esquemas de demostración deductivo con miras a mejorar sus definiciones, construir significados más robustos y profundizar teóricamente sus resultados.

En cada una de las fases planteadas en el diseño de las secuencias de aprendizaje, los estudiantes constantemente están razonando acerca de sus afirmaciones y sus consecuencias. Considerando lo anterior, se evidenció que existe una relación notable entre los tipos de razonamiento utilizados y su correspondencia con los procesos de formulación de la conjetura y el camino hacia la construcción de la demostración.

El razonamiento abductivo actúa como un elemento mediador entre el razonamiento inductivo y el deductivo, que guía el camino desde la formulación de la conjetura hasta la construcción de la demostración matemática. Los estudiantes, llevados por su intuición, el descubrimiento y la observación de lo que sucede en la interacción con las situaciones matemáticas, encuentran fenómenos sorprendentes y desean comprender la razón que los origina. En este punto, como se manifestó antes, los estudiantes razonan hacia atrás tratando de buscar evidencia que corrobore sus hallazgos. Al proceder de esta manera su razonamiento es de tipo inductivo y su objetivo es determinar, clasificar y organizar las pruebas que sustentan sus afirmaciones.

Figura 5. Caracterización del razonamiento abductivo.

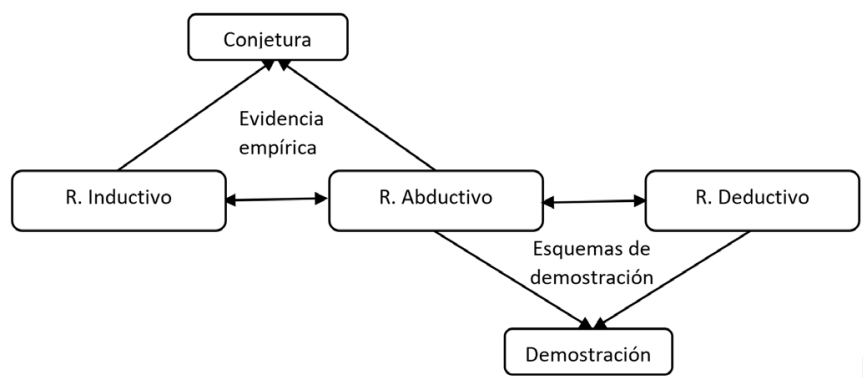

Fuente: elaboración propia 
La manera en que recolectan la información es inductiva y lo hacen a través de estrategias heurísticas tales como tablas de datos, gráficos, análisis de patrones (por recurrencia o por repetición), ejemplos, etc. En este ciclo los estudiantes comienzan a desarrollar esquemas de demostración empíricos que conducen a la formulación y el planteamiento de la conjetura. Una vez identificadas las condiciones que generaron el hecho sorprendente, sus razonamientos cambian de dirección (hacia delante) y se realizan de forma deductiva mediante reglas de inferencia. Ellos reafirman o validan sus resultados mediante la implementación de ejemplos genéricos, problemas semejantes, análisis de casos, métodos exhaustivos, etc.

Este proceso de ir hacia atrás en busca de explicaciones y devolverse de manera deductiva está mediado por el razonamiento abductivo y su propósito es el de encontrar el mejor argumento que dé explicación a lo encontrado. Una vez identificado y validado el argumento, los estudiantes proceden a construir la demostración matemática, sus razonamientos abductivos y deductivos están encaminados a buscar argumentos convencionales o no convencionales (ingeniosos) que conduzcan a la generalización de sus resultados.

Al analizar la forma como los estudiantes construyen sus demostraciones se evidenció que este proceso se da través de la construcción de esquemas de demostración empíricos, deductivos y deductivos transformacionales, siendo los dos últimos los de más aceptación hacia la validez de los resultados.

Este ciclo determinado por la abducción, la deducción y el desarrollo de esquemas de demostración deductivos dan como resultado la demostración matemática, proceso que, en el marco de la resolución de problemas, viene acompañado implícitamente de otros que favorecen y enriquecen el desarrollo del pensamiento matemático del estudiante. En la fase final, los estudiantes no sólo llegaron a la generalización de los resultados mediante el desarrollo de esquemas de demostración, sino que también adoptaron un lenguaje y una notación matemática como una necesidad para 
comunicar sus resultados; este proceso se dio de manera natural a través del pensamiento variacional. El recorrido hecho por los estudiantes desde la fase de exploración hasta la fase de demostración dio origen a la construcción del significado robusto de algunos conceptos (congruencias, algoritmo de la división, múltiplos, divisores, etc.) y su sistematización teórica. Es decir, en la mayoría de los problemas retadores los estudiantes encontraron nuevas proposiciones demostrables y las organizaron desde su concepción, en un nuevo marco teórico.

En la fase de demostración, se observó que el estudiante concibe este proceso como un ritual por estricta apariencia y supone que debe estar aislado de los resultados encontrados en su fase de exploración y validación. Para ellos la demostración debe respetar la apariencia y carácter formal y, aunque todos lograron llegar a la generalización de los problemas retadores y demostraron cada afirmación, al comienzo les fue difícil relacionar sus resultados con la fase final de demostración.

Esta limitación se da por el sistema de creencias que los estudiantes manifiestan alrededor de la demostración como un producto. Una vez identificada esta distorsión dentro de la ID se procede a interactuar y discutir con los estudiantes, a través del recurso de videoconferencia, la funcionalidad de la demostración en el aula desde la concepción establecida en el marco teórico de esta investigación. Se llegó a la conclusión de que la demostración era el resultado de todo el andamiaje utilizado por ellos para llegar a la generalización de las situaciones matemáticas y que en esta fase se culmina todo el proceso mediante la organización y sistematización teórica. No obstante, se evidencia en el estudiante 2 un reconocimiento parcial de este proceso; aunque manifiesta estar de acuerdo con los procesos establecidos en las tres fases, es de gran relevancia mostrar los resultados mediante una apariencia de carácter formal mediante la axiomatización de los resultados. 


\section{Hipótesis global}

La conjetura y demostración matemática en el marco de la resolución de problemas retadores favorecen el desarrollo del pensamiento matemático en los estudiantes de la Licenciatura en Educación Básica con énfasis en Matemáticas de la Universidad Santo Tomás en la modalidad a distancia.

Esta investigación se basó en la implementación positiva de los procesos de conjetura y demostración en el aula y su diseño se abordó desde diferentes aspectos como lo son el contexto, el componente epistemológico, el pedagógico, el didáctico y el matemático. En consecuencia, su análisis mostró una profundización teórica en torno al favorecimiento del pensamiento matemático manifestado por los estudiantes de la Licenciatura en Educación Básica con énfasis en Matemáticas y un modelo didáctico de cómo abordar estos procesos en el aula desde la teoría de números, con miras a modificar desde el pregrado su pensamiento subjetivo en torno a la visión formalista de la demostración matemática.

El diseño de las secuencias de aprendizaje en el marco de la resolución de problemas retadores se convierte en un escenario óptimo para el desarrollo de heurísticas y habilidades matemáticas que conducen al favorecimiento del pensamiento matemático. En cada una de las fases de exploración, validación y demostración dentro del diseño de las secuencias de aprendizaje de la ID, se evidenció por parte de los estudiantes el uso, el progreso y la potencialización de razonamientos y estrategias ingeniosas que los condujeron no solo a resolver las situaciones planteadas, sino a fomentar positivamente procesos de conjetura y demostración en el aula.

En la fase de exploración los estudiantes, mediante la interacción con los objetos, desarrollaron habilidades enfocadas a la comprensión y apropiación de las situaciones matemáticas. El análisis de datos en esta etapa refleja un nivel de comprensión de los estudiantes basado en la manipulación de operaciones concretas. En ellas, sus actuaciones son producto de un análisis experimental, es- 
timulado por mecanismos de ensayo y error y dirigido al reconocimiento de las condiciones establecidas en cada problema retador. Una vez el estudiante se haya situado en el nivel de comprensión del problema sus actuaciones futuras están determinadas por un nivel de apropiación de las situaciones matemáticas, y aunque todavía sus actuaciones están basadas en operaciones concretas sus decisiones están premeditadas por una planificación producto de la exploración, la observación y la reflexión. A medida que el estudiante interactúa y toma control de cada situación matemática, sus razonamientos son más directos y se enfocan más en la observación de hechos sorprendentes y en la recolección de evidencias para el planteamiento de hipótesis y la formulación de conjeturas.

Una vez formulada la conjetura, sus razonamientos y estrategias matemáticas están enfocadas en validar sus afirmaciones. En esta fase hay un desprendimiento de los objetos concretos, y sus explicaciones están basadas en la argumentación, la abstracción y la generalización de las propiedades invariantes de cada una de las situaciones matemáticas. En la fase de validación, las habilidades matemáticas desarrolladas están dirigidas a la verificación o refutación de la conjetura y a la resolución de los problemas retadores; sus argumentos son de carácter abductivo y deductivo, la justificación de sus afirmaciones está condicionada por una secuencia de implicaciones lógicas y por ende sus razonamientos son más refinados y enriquecidos. En este punto el lenguaje matemático es introducido de manera natural por la necesidad de comunicar lo encontrado.

La fase de demostración no debe ser situada como un producto, sino como el resultado de un proceso: no se trata de mostrar el valor asignado solo a la demostración, sino también mostrar de forma organizada y sistemática todos los resultados inmersos que se derivaron del camino de la formulación de la conjetura hasta la etapa de demostración. Al tener en cuenta todo el proceso, el producto final no es la demostración en sí; por el contrario, debe ser el desarrollo del pensamiento matemático implícito en el análisis y solución de las situaciones matemáticas. Este se manifiesta en los cambios conceptuales a nivel cognitivo, pedagógico y didáctico 
provocados en el estudiante durante el desarrollo de las secuencias de aprendizaje, en la profundización teórica de sus hallazgos, en la construcción de significados más robustos, en el refinamiento de sus razonamientos y en el desarrollo de heurísticas cada vez más ingeniosas. Es importante resaltar cómo el análisis de situaciones matemáticas, partiendo desde un conocimiento informal y llevado por una motivación natural de los problemas retadores, despertó en cada estudiante un impulso a querer dar explicación del origen de sus hallazgos hasta llevarlos a un análisis más profundo y original. Sus interacciones constantes en cada problema los condujeron a desarrollar diferentes niveles de pensamiento matemático, desde un nivel básico determinado por el dominio informal de los conceptos matemáticos hasta indagar propiedades y relaciones matemáticas con un nivel de complejidad coherente con el dominio matemático adquirido en cada secuencia de aprendizaje.

\section{Resultados}

Los resultados de este trabajo derivan en un modelo didáctico de formación docente que orienta la manera en que deben implementarse los procesos de conjetura y demostración en el aula desde una perspectiva falibilista. Este modelo didáctico describe los componentes que los docentes deben tener en cuenta al momento de planificar e implementar los procesos de conjetura y demostración en el aula. Es importante resaltar el alcance de esta propuesta ya que está dirigida a docentes en formación cuyo campo profesional se encuentra en los niveles de educación de básica primaria y básica secundaria (sexto a noveno). También es importante tener en cuenta que, aunque el campo de acción de esta investigación fue la formación de docentes en la modalidad a distancia, el alcance del modelo trasciende más allá de los límites de esta modalidad, debido a que sus componentes están determinados por las relaciones implícitas en los procesos de conjetura y demostración matemática descritos en cada una de sus fases (exploración, validación y demostración) y no en las características de la educación a distancia. 
Figura 6. Modelo didáctico de la implementación de la conjetura y demostración en el aula.

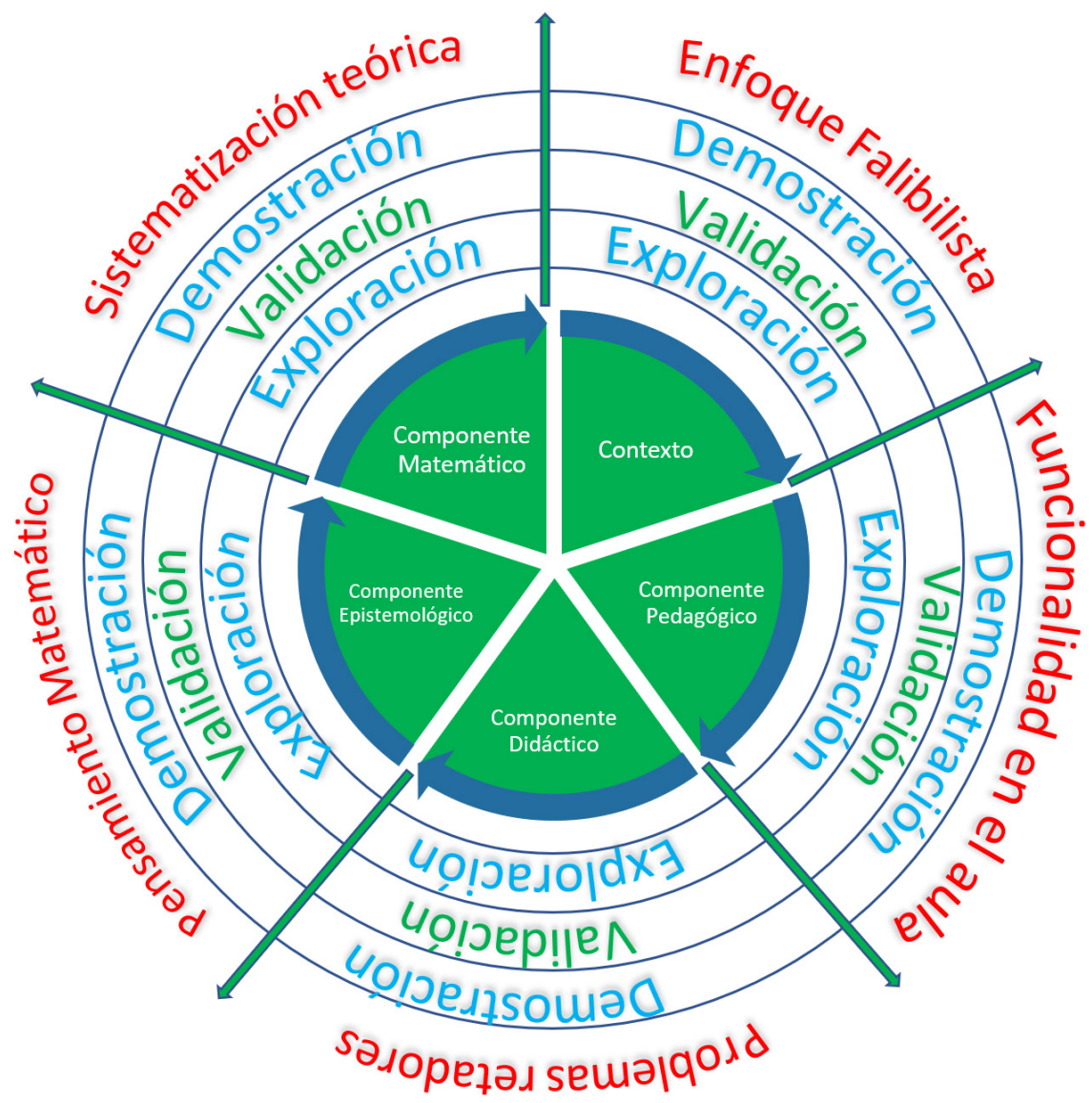

Fuente: elaboración propia

Para la planificación e implementación de los procesos de conjetura y demostración en el aula se deben tener en cuenta componentes más allá de los aspectos matemáticos de la demostración. No sólo es importante hacer énfasis en el conocimiento matemático y los estándares que debe cumplir la demostración, sino que esta se debe abordar teniendo en cuenta el contexto en que se producen. El contexto está determinado por las creencias de los maestros, los aspectos históricos de la demostración y el currículo. Las creencias determinan el enfoque del maestro al momento de planificar e implementar la conjetura y demostración en el aula. Si el maestro tiene un bagaje histórico de los procesos de conjetura y 
demostración desde los babilonios hasta nuestros días, sus creencias estarán direccionadas hacia un enfoque falibilista y ajustará el currículo teniendo en cuenta la población que atenderá.

Una vez identificado el contexto, el docente debe planificar sus actividades de acuerdo con los componentes pedagógicos y didácticos de la conjetura y demostración en el aula, es decir, es importante que el docente tenga claridad sobre el para qué y el cómo de la implementación de estos procesos. El para qué hace referencia a la funcionalidad de la demostración y el cómo a la forma de abordarla, que en esta investigación se hizo desde el marco de la resolución de problemas. El componente epistemológico está dirigido hacia el desarrollo del pensamiento matemático en los estudiantes: no solamente debe demostrarse el valor de verdad de una afirmación, sino que también es importante potencializar el razonamiento matemático implícito en cada demostración y el estudio del pensamiento matemático en cada proceso manifestado por los estudiantes. El componente matemático debe estar orientado hacia la sistematización teórica de los resultados y hacia la construcción de significados más robustos, y se deja al final para resaltar que los procesos de conjetura y demostración en el aula no deben atender solamente a los contenidos matemáticos inmersos en una demostración. Cada uno de los componentes del modelo están conectados, de tal forma que la planificación de una nueva actividad debe respetar este ciclo secuencial.

Una vez se establezca el diseño de las secuencias de aprendizaje, las fases de exploración, validación y demostración determinan el alcance de los resultados en los procesos de conjetura y demostración. Este alcance se mide mediante la interacción constante de los estudiantes con las secuencias de aprendizaje. El resultado final es un desarrollo creciente y expandible de todos los componentes. A continuación, se muestra la caracterización del proceso de conjetura y demostración matemática en el aula manifestada por los estudiantes de la Licenciatura en Educación Básica con énfasis en Matemáticas en la modalidad a distancia en la asignatura de Número y Cantidad. 
Figura 7. Caracterización de los procesos de conjetura y demostración matemática manifestada por los docentes en formación.

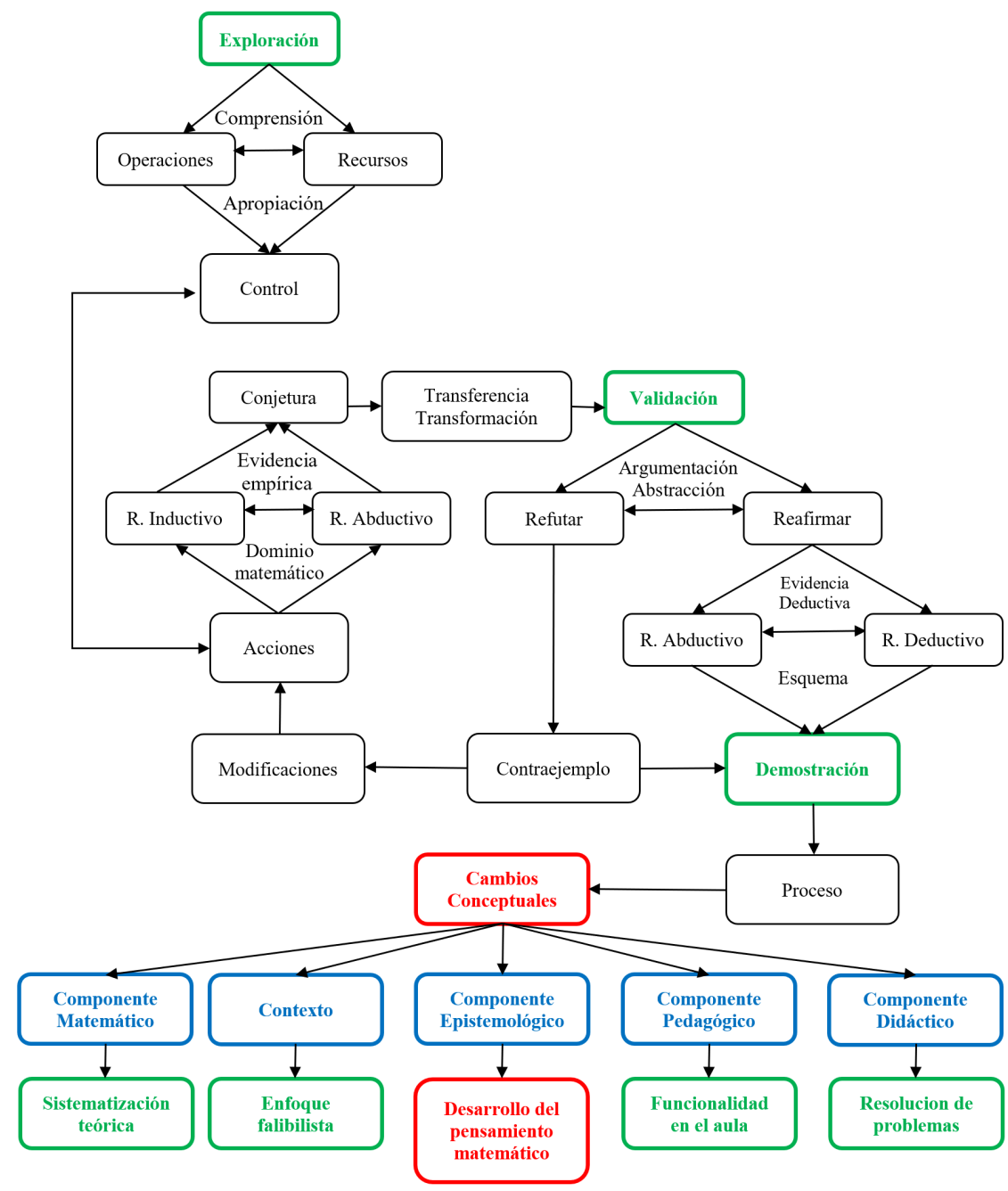

Fuente: elaboración propia

Cada proceso inmerso en las fases de exploración, validación y demostración fue previamente explicado y detallado en el análisis a posteriori y validación de los resultados. 


\section{Conclusiones}

Los resultados de esta investigación en los procesos de conjetura y demostración desde una perspectiva falibilista generaron cambios conceptuales en los componentes matemático, epistemológico, pedagógico y didáctico. Por tanto, se hace necesario seguir avanzando en investigaciones que respalden los alcances de implementar prácticas positivas en el aula desde los primeros niveles de formación.

Si se define el camino desde los primeros años para que los estudiantes de manera natural se enfrenten con escenarios donde la demostración matemática tenga el sentido y la importancia que se merece, muy seguramente en los siguientes niveles de formación los esquemas que desarrollen los estudiantes se refinarán a tal punto que sus razonamientos estarán determinados por una estructura deductiva y de carácter axiomática paralela a la que desarrollan los matemáticos.

Fuera del campo de los matemáticos, profesores de matemáticas y estudiantes con un buen dominio matemático, los procesos de demostración en el aula carecen de sentido. Esta investigación está dirigida a fortalecer los procesos de conjetura y demostración en el aula a estudiantes y docentes inexpertos cuyo dominio matemático es mínimo pero que motivados por problemas retadores logran desarrollar esquemas de demostración deductivos. Teniendo en cuenta que la población en Colombia, como lo señalan las pruebas internacionales, presenta deficiencias en matemáticas, se recomienda seguir investigando en el diseño de secuencias de aprendizaje basadas en el enfoque de resolución de problemas retadores con miras a construir un conjunto de prácticas que consoliden un material didáctico y pedagógico que sea base para la formación de docentes. 


\section{Referencias bibliográficas}

Artigue, M., Douady, R., Moreno, L. y Gómez, P. (1995). Ingeniería didáctica en educación matemática. Bogotá.

de Losada, M. F. (2001). Olimpiadas de Matemáticas: retos, logros (y frustraciones). Boletín de la Asociación Matemática Venezolana, 8(1), 15-26.

Hanna, G. (2000). Proof, Explanation and Exploration: An Overview. Educational Studies in Mathematics, 44(1), 5-23.

Harel, G. y Sowder, L. (2007). Toward Comprehensive Perspectives on the Learning and Teaching of Proof. En F. Lester, Jr. (ed.), Second Handbook of Research on Mathematics Teaching and Learning (vol. 2, pp. 805842). USA: IAP.

Harel, G., Stylianides, A. J., Boero, P., Miyazaki, M. y Reid, D. (2017). Topic Study Group No. 18: Reasoning and Proof in Mathematics Education. En G. Kaiser (ed.), Proceedings of the 13th International Congress on Mathematical Education (pp. 459-461). Cham: Springer.

Neiman, G. y Quaranta, G. (2012). Los estudios de caso en la investigación cualitativa. Estrategias de investigación cualitativa. Barcelona: Gedisa.

Petrou, M., \& Goulding, M. (2011). Conceptualising teachers' mathematical knowledge in teaching. In Mathematical knowledge in teaching (pp. 9-25). Springer, Dordrecht.

Schoenfeld, A. H. (1985). Mathematical problem solving. New York: Academic Press.

Strauss, A. L. y Corbin, J. (2002). Bases de la investigación cualitativa: técnicas y procedimientos para desarrollar la teoría fundamentada (trad. E. Zimmerman). Medellín: Universidad de Antioquia. 
Stylianides, G. J. y Stylianides, A. J. (2017). Based Interventions in the Area of Proof: the Past, the Present, and the Future. Educational Studies in Mathematics, 96(2), 119-127.

Toscano, F. S. (2012). Razonamiento abductivo en lógica clásica. College Publications.

Zazkis, R., \& Campbell, S. R. (2006). Number Theory in mathematics education research: perspectives and prospects. Number Theory in mathematics education: Perspectives and prospects, 1-18.

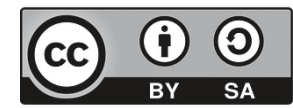

